\title{
Marginally bound circular orbits in the composed black-hole-ring system
}

\author{
Shahar Hod ${ }^{1,2, a}$ \\ ${ }^{1}$ The Ruppin Academic Center, 40250 Emeq Hefer, Israel \\ 2 The Hadassah Academic College, 91010 Jerusalem, Israel
}

Received: 8 September 2020 / Accepted: 6 October 2020 / Published online: 14 October 2020

(C) The Author(s) 2020

\begin{abstract}
The physical and mathematical properties of the non-linearly coupled black-hole-orbiting-ring system are studied analytically to second order in the dimensionless angular velocity $M_{\mathrm{ir}} \omega_{\mathrm{H}}$ of the black-hole horizon (here $M_{\mathrm{ir}}$ is the irreducible mass of the slowly rotating central black hole). In particular, we determine analytically, to first order in the dimensionless ring-to-black-hole mass ratio $m / M_{\text {ir }}$, the shift $\Delta \Omega_{\mathrm{mb}} / \Omega_{\mathrm{mb}}$ in the orbital frequency of the marginally bound circular geodesic that characterizes the composed curved spacetime. Interestingly, our analytical results for the frequency shift $\Delta \Omega_{\mathrm{mb}}$ in the composed black-hole-orbiting-ring toy model agree qualitatively with the recently published numerical results for the corresponding frequency shift in the physically related (and mathematically much more complex) black-hole-orbiting-particle system. In particular, the present analysis provides evidence that, at order $O\left(m / M_{\text {ir }}\right)$, the recently observed positive shift in the angular frequency of the marginally bound circular orbit is directly related to the physically intriguing phenomenon of dragging of inertial frames by orbiting masses in general relativity.
\end{abstract}

\section{Introduction}

Geodesic orbits of test particles in curved spacetimes are of central importance in black-hole physics [1-14]. They provide valuable information on the physical parameters (mass, charge, angular momentum) of the central black hole. In particular, the marginally bound circular orbit of a curved blackhole spacetime is of special importance in astrophysics and general relativity [1-14]. This physically interesting geodesic represents the innermost circular orbit of a massive particle which is energetically bound to the central black hole.

\footnotetext{
a e-mail: shaharhod@gmail.com (corresponding author)
}

For a test particle of proper mass $m$, the marginally bound circular geodesic is characterized by the simple energy relation [1-4]

$E\left(r_{\mathrm{mb}}\right)=m$,

where $E$ is the energy of the particle as measured by asymptotic observers. Interestingly, the marginally bound circular geodesic (1) marks the boundary between bound orbits, which are characterized by the sub-critical energy relation $E<m$, and unbound circular orbits with $E>m$ which, given a small outward perturbation, can escape to infinity. In particular, as nicely demonstrated in $[5,6]$, the critical (marginally bound) circular geodesic (1) plays a key role in the dynamics of star clusters around super-massive black holes in galactic nuclei [The critical orbit (1) is sometimes referred to in the physics literature as the innermost bound spherical orbit (IBSO) [6,7]].

An important gauge-invariant physical quantity that characterizes the motion of particles along the marginally bound circular geodesic is the orbital angular frequency $\Omega_{\mathrm{mb}}$ of the particles as measured by asymptotic observers. For a test-particle moving in the spinless spherically symmetric Schwarzschild black-hole spacetime, this physically important orbital frequency is given by the simple dimensionless relation [2-4]

$M_{\mathrm{ir}} \Omega_{\mathrm{mb}}=\frac{1}{8}$.

Here $M_{\text {ir }}$ is the irreducible mass [15] of the central black hole.

In recent years, there is a growing physical interest in calculating the $O\left(m / M_{\text {ir }}\right)$ corrections to the orbital frequency (1) of the marginally bound circular orbit in non-linearly coupled black-hole-particle systems (see the physically interesting work [16] and references therein). To this end, one should 
take into account the gravitational self-force corrections to the geodesic orbits of the particles [17-33].

The gravitational self-force has two distinct physical contributions to the dynamics of a composed black-hole-particle system:

(1) It is responsible for non-conservative physical effects, like the emission of energy and angular momentum in the form of gravitational waves $[17,18]$.

(2) The composed black-hole-particle system is also characterized by conservative gravitational self-force effects that preserve the total energy and angular momentum of the system but shift the orbital frequency of the marginally bound orbit.

Computing the gravitational self-force (GSF) correction $\Delta \Omega_{\mathrm{mb}}$ to the zeroth-order frequency (1) of the marginally bound circular orbit is a highly non-trivial task. Intriguingly, Barack et al. [16] have recently used sophisticated numerical techniques in the composed Schwarzschild-black-holeorbiting-particle system in order to compute the characteristic shift $\Delta \Omega_{\mathrm{mb}}$ in the orbital frequency of the marginally bound orbit which is caused by the conservative part of the GSF.

In particular, Barack et al. [16] have found the (positive) dimensionless value

$$
\frac{\Delta \Omega_{\mathrm{mb}}}{\Omega_{\mathrm{mb}}}=c \cdot \eta+O\left(\eta^{2}\right) \quad \text { with } c \simeq 0.5536
$$

for the shift in the orbital frequency of the marginally bound circular orbit, where

$\eta \equiv \frac{m}{M_{\mathrm{ir}}}$

is the dimensionless ratio between the mass of the orbiting particle and the mass of the central Schwarzschild black hole. The physical importance of the result (3) of [16] stems from the fact that it provides gauge-invariant information about the strong-gravity effects in the highly curved region $\left(r \simeq 4 M_{\text {ir }}\right)$ of the black-hole spacetime.

The main goal of the present compact paper is to use analytical techniques in order to gain some physical insights on the intriguing $O\left(m / M_{\mathrm{ir}}\right)$ increase [see Eq. (3)] in the orbital frequency of the marginally bound circular orbit as recently observed numerically in the physically important work [16]. In particular, we shall analyze a simple black-hole-orbitingring toy model which, as we shall explicitly show below, captures some of the essential physical features of the (astrophysically more interesting and mathematically much more complex) black-hole-orbiting-particle system in general relativity. As nicely proved by Will [34,35], the composed blackhole-orbiting-ring toy model is amenable to a perturbative analytical treatment to second order in the dimensionless angular velocity $M_{\mathrm{ir}} \omega_{\mathrm{H}}$ of the central slowly rotating black hole.

\section{The orbital frequency of the marginally bound circular orbit in the composed black-hole-orbiting-ring spacetime}

In the present paper we would like to gain some analytical insights into the conservative part of the $O(\mathrm{~m} / \mathrm{M})$-shift in the orbital frequency $\Omega_{\mathrm{mb}}$ of the marginally bound orbit that has recently been computed numerically in the highly interesting work [16]. To this end, we shall use the analytically solvable model of an axisymmetric ring of matter which orbits a central slowly spinning black hole [34,35]. In particular, we shall use this simplified axisymmetric toy model (which, due to its symmetry, has no dissipative effects) in order to model the conservative part of the dynamics of the mathematically more complex black-hole-orbiting-particle system [36].

We expect the composed axisymmetric black-hole-orbitingring system to capture, at least qualitatively, the essential physical features that characterize the conservative dynamics of the composed black-hole-orbiting-particle system. In particular, both the orbiting particle in the black-hole-particle system and the orbiting ring in the black-hole-ring system drag the generators of the central black-hole horizon $[34,35]$.

The physically intriguing general relativistic effect of dragging of inertial frames by an orbiting object is reflected, both in the black-hole-particle system and in the black-holering system, by a non-linear spin-orbit interaction term of order $\omega_{\mathrm{H}} \cdot j$ in the total gravitational energy of the composed systems (here $\omega_{\mathrm{H}}$ is the angular velocity of the black-hole horizon and $j$ is the angular momentum per unit mass of the orbiting ring).

Interestingly, and most importantly for our analysis, the main mathematical advantage of the black-hole-orbitingring system over the physically more interesting (but mathematically more complex) black-hole-orbiting-particle system stems from the fact that the spin-orbit interaction term in the black-hole-ring system is known in a closed analytical form to second order in the dimensionless angular velocity $M_{\text {ir }} \omega_{\mathrm{H}}$ of the central black hole $[34,35]$ [see Eq. (10) below].

In a very interesting work, Will $[34,35]$ has analyzed the total gravitational energy and the total angular momentum of a stationary physical system which is composed of an axisymmetric ring of particles of proper mass $m$ which orbits a central slowly rotating black hole of an irreducible mass $M_{\text {ir. }}$ In particular, it has been proved in [34,35] that the composed axisymmetric black-hole-orbiting-ring system is characterized by the total angular momentum

$$
J_{\text {total }}(x)=m j+4 M_{\mathrm{ir}}^{3} \omega_{\mathrm{H}}-8 m j x^{3},
$$


where

$x \equiv \frac{M_{\mathrm{ir}}}{R}$

is the dimensionless ratio between the irreducible mass of the black hole and the proper circumferential radius of the ring,

$j(x)=\frac{M_{\mathrm{ir}}}{[x(1-3 x)]^{1 / 2}} \cdot\left[1+O\left(M_{\mathrm{ir}} \omega_{\mathrm{H}}\right)\right]$

is the angular momentum per unit mass of the orbiting ring, and $\omega_{\mathrm{H}}$ is the angular velocity of the black-hole horizon.

Since the first term on the r.h.s. of (5) represents the angular momentum $J_{\text {ring }}$ of the orbiting ring of mass $m$, one concludes $[34,35]$ that the last two terms in (5) represent the angular momentum

$J_{\mathrm{H}}=4 M_{\mathrm{ir}}^{3} \omega_{\mathrm{H}}-8 m j x^{3}$

which is contributed by the slowly spinning central black hole as measured by asymptotic observers. In particular, it is interesting to point out that, while the first term in (8) represents the usual relation between the angular momentum and the angular velocity of a slowly rotating Kerr black hole, the second term on the r.h.s. of (8) is a direct consequence of the dragging of inertial frames caused by the orbiting ring $[34,35]$.

A simple inspection of the compact expression (8) reveals the physically important fact that, unlike vacuum Schwarzschild black holes, a zero angular momentum $\left(J_{\mathrm{H}}=\right.$ 0 ) black hole in the non-linearly coupled black-hole-orbitingring system is characterized by the non-zero horizon angular velocity

$\omega_{\mathrm{H}}\left(J_{\mathrm{H}}=0\right)=\frac{2 x^{3}}{M_{\mathrm{ir}}^{3}} \cdot m j$.

In addition, it has been explicitly proved in $[34,35]$ that, to second order in the angular velocity of the black-hole horizon, the composed axisymmetric black-hole-orbiting-ring system is characterized by the total gravitational energy

$$
\begin{aligned}
E_{\mathrm{total}}(x)= & m-m \Phi(x)+M_{\mathrm{ir}}+2 M_{\mathrm{ir}}^{3} \omega_{\mathrm{H}}^{2}-\omega_{\mathrm{H}} m j \Psi(x) \\
& -\frac{m^{2} x}{2 \pi M_{\mathrm{ir}}} \ln \left(\frac{8 M_{\mathrm{ir}}}{x r}\right)
\end{aligned}
$$

as measured by asymptotic observers. Here we have used the dimensionless radially dependent functions

$$
\Phi(x) \equiv 1-\frac{1-2 x}{(1-3 x)^{1 / 2}} ; \quad \Psi(x) \equiv 12 \frac{x^{3}-2 x^{4}}{1-3 x}
$$

The various terms in the energy expression (10), which characterizes the composed black-hole-orbiting-ring system, have the following physical interpretations $[34,35]$ :

- The first term in the energy expression (10) represents the proper mass of the ring.

- In order to understand the physical meaning of the second term in the energy expression (10), it is worth pointing out that, in the small- $x$ regime (large ring radius, $R \gg M_{\text {ir }}$ ), this term can be approximated by the compact expression [see Eqs. (6), (10), and (11)] $-M_{\mathrm{ir}} m / 2 R \cdot\left[1+O\left(M_{\mathrm{ir}} / R\right)\right]$, which is simply the sum of the potential and rotational Newtonian energies of the ring in the background of the central compact object. Thus, this term represents the leading order (linear in the mass $m$ of the ring) interaction between the central black hole and the orbiting ring.

- In order to understand the physical meaning of the third and fourth terms in the energy expression (10), it is worth pointing out that a slowly spinning bare (isolated) Kerr black hole is characterized by the simple mass-angularvelocity relation $M_{\mathrm{Kerr}}=M_{\mathrm{ir}}+2 M_{\mathrm{ir}}^{3} \omega_{\mathrm{H}}^{2}+O\left(M_{\mathrm{ir}}^{5} \omega_{\mathrm{H}}^{4}\right)$. Thus, the third and fourth terms in (10) can be identified as the contribution of the slowly spinning central black hole to the total energy of the system. Interestingly, taking cognizance of Eq. (9) one learns that due to the general relativistic frame dragging effect, which is caused by the orbital motion of the ring, the fourth term in (10) contains a self-interaction contribution [of order $O\left(\mathrm{~m}^{2} / M_{\text {ir }}\right)$ ] to the total energy of the composed black-hole-orbiting-ring system.

- The fifth term in the energy expression (10) is a non-linear spin-orbit interaction between the slowly spinning central black hole and the orbiting ring. This energy term plays a key role in our composed black-hole-orbitingring toy model system since it is expected to mimic, at least qualitatively, the physically analogous nonlinear spin-orbit interaction in the original black-holeorbiting-particle system. Taking cognizance of Eq. (9) one learns that, due to the intriguing general relativistic phenomenon of frame dragging, the spin-orbit interaction term in (10) contains a non-linear contribution to the total energy of the composed black-hole-orbiting-ring system which is of order $O\left(\mathrm{~m}^{2} / M_{\mathrm{ir}}\right)$.

- The sixth term in the energy expression (10) is the gravitational self-energy of the ring [37] (not discussed in $[34,35])$, where $r \ll R$ is the half-thickness of the ring. This energy contribution represents the inner interactions between the many particles that compose the axisymmetric ring. Since our main goal in the present paper is to present a simple analytical toy-model for the physically more interesting (and mathematically more complex) two-body system in general relativity, which is characterized by a single orbiting particle, we shall not con- 
sider here this many-particle energy contribution. This approximation allows one to focus the physical attention on the general relativistic frame-dragging effect which characterizes both the black-hole-orbiting-particle system and the black-hole-orbiting-ring system.

Taking cognizance of Eqs. (7) and (9)-(11), one finds the compact functional expression

$$
\begin{aligned}
E_{\text {total }}(x)= & M_{\text {ir }}+m \cdot\left[\frac{1-2 x}{(1-3 x)^{1 / 2}}\right. \\
& \left.+\frac{8 x^{5}(-2+3 x)}{(1-3 x)^{2}} \cdot \eta+O\left(\eta^{2}\right)\right]
\end{aligned}
$$

for the total gravitational energy of the non-linearly coupled black-hole-orbiting-ring system.

In the decoupling $R / M_{\mathrm{ir}} \rightarrow \infty$ limit, in which the ring is located at spatial infinity, the system is characterized by the presence of two non-interacting physical objects: (1) a bare (unperturbed) Schwarzschild black hole of mass $M=M_{\mathrm{ir}}$ [38], and (2) a ring of proper mass $m$. Thus, the total energy of the black-hole-ring system in the $R / M_{\text {ir }} \rightarrow \infty$ limit is given by the simple expression [see Eq. (12) with $x \rightarrow 0$ ]

$E_{\text {total }}\left(R / M_{\text {ir }} \rightarrow \infty\right)=M+m$ with $M=M_{\text {ir }}$.

Energy conservation implies that the marginally bound orbit of the composed black-hole-orbiting-ring system is characterized by the same total gravitational energy [39]

$E_{\text {total }}\left(x=x_{\mathrm{mb}}\right)=M_{\text {ir }}+m$

as measured by asymptotic observers. Substituting the relation (14) into Eq. (12), one finds the simple expression

$x_{\mathrm{mb}}=\frac{1}{4} \cdot\left[1+\frac{5}{16} \cdot \eta+O\left(\eta^{2}\right)\right]$

for the $O\left(m / M_{\mathrm{ir}}\right)$-corrected location of the marginally bound circular orbit in the composed black-hole-orbiting-ring system.

Substituting the dimensionless radial coordinate (15) of the marginally bound orbit into the functional expression [34, 35]

$$
M_{\mathrm{ir}} \Omega=x^{3 / 2} \cdot\left[1-4 x^{3 / 2} \cdot M_{\mathrm{ir}} \omega_{\mathrm{H}}+O\left[\left(M_{\mathrm{ir}} \omega_{\mathrm{H}}\right)^{2}\right]\right]
$$

for the dimensionless orbital frequency of the axisymmetric orbiting ring and using Eqs. (7) and (9) [40], one obtains the $O\left(m / M_{\text {ir }}\right)$-corrected expression
$M_{\mathrm{ir}} \Omega_{\mathrm{mb}}=\frac{1}{8} \cdot\left[1+\frac{13}{32} \cdot \eta+O\left(\eta^{2}\right)\right]$

for the characteristic orbital frequency of the marginally bound circular geodesic in the composed black-hole-orbitingring system.

\section{Summary}

We have analyzed the physical and mathematical properties of a composed black-hole-orbiting-ring system. In particular, we have proposed to use this analytically solvable conservative [36] system as a simple toy model for the conserved dynamics of the astrophysically more interesting (and mathematically more complex) black-hole-orbiting-particle system in general relativity.

Our main goal was to provide a simple qualitative analytical explanation for the increase in the orbital frequency of the marginally bound circular geodesic that has recently been observed numerically in the physically important work [16]. To this end, we have used the non-trivial spin-orbit interaction between the central black hole and the orbiting ring, which is known in a closed analytical form to second order in the dimensionless angular velocity $M_{\mathrm{ir}} \omega_{\mathrm{H}}$ of the black-hole horizon, in order to capture the essential physical features of a similar non-linear spin-orbit interaction which is expected to characterize the conservative dynamics of the black-hole-orbiting-particle system.

Interestingly, the analytically derived expression [see Eqs. (2) and (17)]

$\frac{\Delta \Omega_{\mathrm{mb}}}{\Omega_{\mathrm{mb}}}=\frac{13}{32} \cdot \eta+O\left(\eta^{2}\right)$

for the dimensionless $O\left(m / M_{\text {ir }}\right)$-shift in the orbital frequency of the marginally bound circular geodesic in the composed black-hole-orbiting-ring system provides the correct order of magnitude (with the correct sign) for the corresponding shift in the orbital frequency of the marginally bound circular geodesic of the physically more interesting black-hole-orbiting-particle system.

This qualitative agreement suggests that the observed shift (3) in the characteristic orbital frequency of the marginally bound circular geodesic is mainly determined by the general relativistic effect of dragging of inertial frames by orbiting objects (the non-linear spin-orbit interaction between the orbiting object and the generators of the central black-hole horizon). 
Acknowledgements This research is supported by the Carmel Science Foundation. I thank Yael Oren, Arbel M. Ongo, Ayelet B. Lata, and Alona B. Tea for stimulating discussions.

Data Availability Statement This manuscript has no associated data or the data will not be deposited. [Authors' comment: I would like to emphasize that all relevant physical and mathematical calculations are explicitly presented in this paper.].

Open Access This article is licensed under a Creative Commons Attribution 4.0 International License, which permits use, sharing, adaptation, distribution and reproduction in any medium or format, as long as you give appropriate credit to the original author(s) and the source, provide a link to the Creative Commons licence, and indicate if changes were made. The images or other third party material in this article are included in the article's Creative Commons licence, unless indicated otherwise in a credit line to the material. If material is not included in the article's Creative Commons licence and your intended use is not permitted by statutory regulation or exceeds the permitted use, you will need to obtain permission directly from the copyright holder. To view a copy of this licence, visit http://creativecomm ons.org/licenses/by/4.0/.

Funded by SCOAP ${ }^{3}$.

\section{References}

1. B. Carter, Phys. Rev. 174, 1559 (1968)

2. J.M. Bardeen, W.H. Press, S.A. Teukolsky, Astrophys. J. 178, 347 (1972)

3. S. Chandrasekhar, The Mathematical Theory of Black Holes (Oxford University Press, New York, 1983)

4. S.L. Shapiro, S.A. Teukolsky, Black Holes, White Dwarfs, and Neutron Stars: The Physics of Compact Objects (Wiley, New York, 1983)

5. D. Merritt, T. Alexander, S. Mikkola, C.M. Will, Phys. Rev. D 84, 044024 (2011)

6. C.M. Will, Class. Quantum Gravity 29, 217001 (2012)

7. R. Grossman, J. Levin, G. Perez-Giz, Phys. Rev. D 85, 023012 (2012)

8. S. Hod, Phys. Rev. D 84, 104024 (2011). arXiv:1201.0068

9. S. Hod, Phys. Rev. D 84, 124030 (2011). arXiv: 1112.3286

10. S. Hod, Phys. Lett. B 718, 1552 (2013). arXiv:1210.2486

11. S. Hod, Phys. Rev. D 87, 024036 (2013). arXiv: 1311.1281

12. S. Hod, Phys. Rev. D 88, 087502 (2013). arXiv: 1707.05680

13. S. Hod, Phys. Lett. B 726, 533 (2013). arXiv:1312.4969

14. S. Hod, Eur. Phys. J. C 74, 2840 (2014). arXiv: 1404.1566

15. The irreducible mass of a black hole is related to its surface area $A$ by the simple relation $M_{\text {ir }}=(A / 16 \pi)^{1 / 2}$. For a spherically symmetric vacuum Schwarzschild black hole, the irreducible mass coincides with the total ADM mass $M$ of the spacetime: $M_{\mathrm{ir}}=M$
16. L. Barack, M. Colleoni, T. Damour, S. Isoyama, N. Sago, Phys. Rev. D 100, 124015 (2019)

17. A. Ori, K.S. Thorne, Phys. Rev. D. 62, 124022 (2000)

18. A. Buonanno, T. Damour, Phys. Rev. D 62, 064015 (2000)

19. E. Poisson, Living Rev. Relativ. 7, 6 (2004)

20. C.O. Lousto, Class. Quantum Gravity 22, S369 (2005)

21. S. Detweiler, in Mass and Motion in General Relativity, ed. by L. Blanchet, A. Spallicci, B. Whiting (Springer, Berlin, 2011)

22. L. Barack, Class. Quantum Gravity 26, 213001 (2009)

23. S. Detweiler, Phys. Rev. D 77, 124026 (2008)

24. N. Sago, L. Barack, S. Detweiler, Phys. Rev. D 78, 124024 (2008)

25. T.S. Keidl, A.G. Shah, J.L. Friedman, D. Kim, L.R. Price, Phys. Rev. D 82, 124012 (2010)

26. A. Shah, T. Keidl, J. Friedman, D. Kim, L. Price, Phys. Rev. D 83, 064018 (2011)

27. T. Damour, Phys. Rev. D 81, 024017 (2010)

28. L. Barack, N. Sago, Phys. Rev. Lett. 102, 191101 (2009)

29. L. Barack, N. Sago, Phys. Rev. D 81, 084021 (2010)

30. S. Akcay, L. Barack, T. Damour, N. Sago, Phys. Rev. D 86, 104041 (2012)

31. M. Favata, Phys. Rev. D 83, 024027 (2011)

32. M. Favata, Phys. Rev. D 83, 024028 (2011)

33. B. Kol, arXiv: 1307.4064

34. C.M. Will, Astrophys. J. 191, 521 (1974)

35. C.M. Will, Astrophys. J. 196, 41 (1975)

36. It is worth stressing the fact that the dynamics of the axisymmetric black-hole-orbiting-ring system is conservative in the sense that, due to its simple symmetry, it contains no gravitational waves. Likewise, the conservative part of the dynamics of the composed black-hole-orbiting-particle system ignores the emission of energy and angular momentum in the form of gravitational waves

37. K.S. Thorne, in Quasi-Stellar Sources and Gravitational Collapse (University of Chicago, Chicago, 1965)

38. Note that a bare Schwarzschild black hole is characterized by the simple relation $M_{\mathrm{ir}}=(A / 16 \pi)^{1 / 2}=M$

39. We consider composed black-hole-orbiting-ring configurations which are characterized by a fixed value of the geometrically important quantity $M_{\text {ir }}$ (that is, a fixed value of the central black-hole surface area)

40. Note that one finds from Eqs. (7) and (9) the dimensionless relation $M_{\mathrm{ir}} \omega_{\mathrm{H}}=\frac{1}{8} \eta \cdot[1+O(\eta)]$ for the angular velocity of the black-hole horizon when the orbiting ring is in its marginally bound orbit (15) 